\title{
Detector Simulation and Reconstruction of Supernova Neutrinos with JUNO
}

\section{Thilo Birkenfeld**}

Physics Institute III B, RWTH Aachen University, Otto-Blumenthal-Straße, Aachen, Germany

E-mail: birkenfeld@physik.rwth-aachen.de

On behalf of the JUNO collaboration

\begin{abstract}
Since the detection of neutrinos emitted by the supernova SN 1987A, no neutrinos from other supernovae have been observed to date. The Jiangmen Underground Neutrino Observatory (JUNO) will be capable of measuring the neutrino burst from a galactic supernova explosion. High statistics, a low detection threshold, and an excellent energy resolution will strongly constrain the details of the neutrino-driven supernova mechanism. JUNO will be sensitive to signals from all neutrino flavors via different detection channels. These are the inverse beta decay, elastic scattering on protons and electrons, and various interactions with carbon. The capability of separating these channels is challenging but crucial for flavor dependent analyses of the supernova burst. We present initial results of an event classification that is based on a full detector simulation.
\end{abstract}

40th International Conference on High Energy physics - ICHEP2020

July 28 - August 6, 2020

Prague, Czech Republic (virtual meeting)

${ }^{*}$ Speaker 


\section{Introduction}

A typical core-collapse supernova $(\mathrm{SN})$ releases $O\left(10^{53} \mathrm{erg}\right)$ of its gravitational energy via the emission of (anti-)neutrinos in all flavors [1]. Detailed insight into the mechanism of core-collapse supernovae could be achieved, if a galactic SN burst is observed by the upcoming generation of neutrino detectors [2]. We present an investigation of the energy spectra reconstruction capability of JUNO [3], including event selection efficiencies (see [4] for previous work).

\section{Data Simulation}

Studying JUNOs response on a galactic supernova burst requires a full Monte Carlo detector simulation, that uses a prediction of the neutrino flux at the JUNO site. The JUNO collaboration provides a full simulation of the detector [5]. The complex evolution of the SN neutrino flux can not be parameterized and predictions require extensive numeric simulations. We use a set of simulations provided by H.-T. Janka et al. [6]. It varies in different parameters (e.g. initial mass, equation of state etc.). A distance of $10 \mathrm{kpc}$ to the $\mathrm{SN}$ is assumed for all simulations.

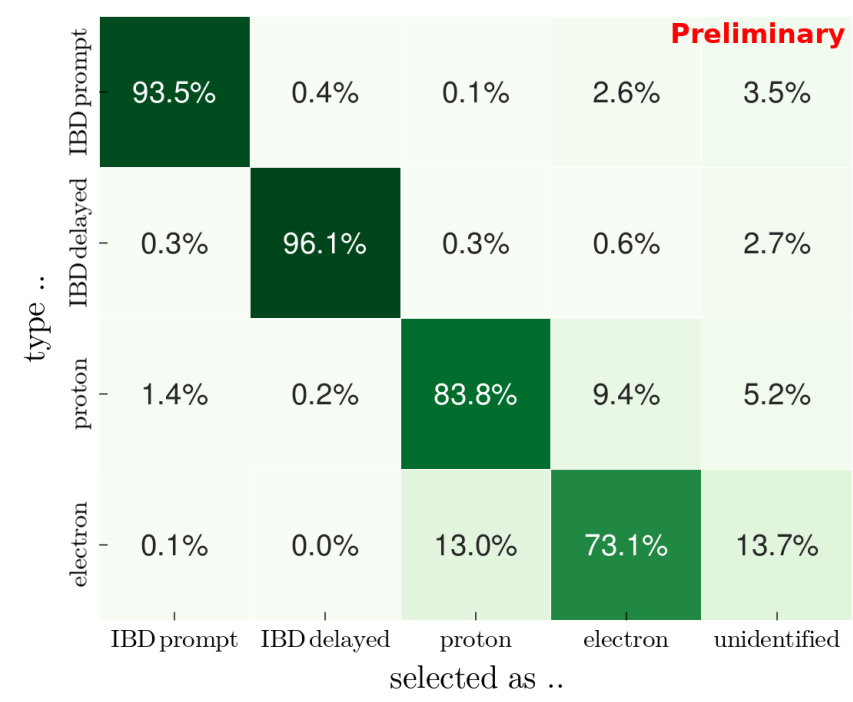

Figure 1: Event selection confusion matrix. Rows denote the true signal type, columns the selected class. Entries are the fractions of the true event types (row) that are selected into the corresponding classes (column). The unidentified class was added for uncertain identifications, caused by overlapping distributions.

\section{Event Selection}

The main interaction channel for electron anti-neutrinos in the $\mathrm{MeV}$ range is the Inverse Beta Decay (IBD) [3, 7]. In liquid scintillator it leads to two signals, a prompt positron and a delayed neutron capture. This delayed coincidence is identified by cuts that rely on the time difference between two triggered events, their spatial distance and energies. The other two main channels are elastic scattering on electrons and protons, for all neutrino flavors [3]. These two channels are separated by an energy cut, as the visible energy of the proton gets heavily quenched [8]. All cuts are optimized for SN bursts and the corresponding confusion matrix is shown in Figure 1. 


\section{Energy Spectrum Unfolding}

The event selection and a visible energy reconstruction is applied to all simulations. Some of these are used to determine the detector response. The energy spectra of the others are reconstructed by applying Iterative Bayesian Unfolding [9]. Preliminary results are shown in Figure 2 for a $27 \mathrm{M}_{\odot}$ core-collapse SN. A flavor dependent unfolding will be the effort of future analysis.

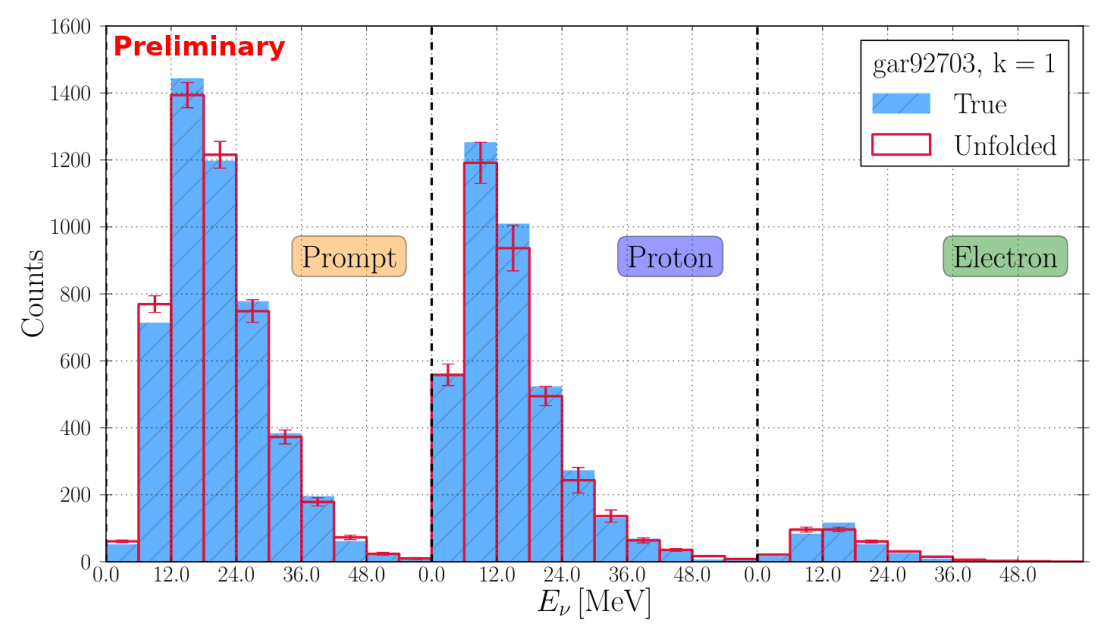

Figure 2: Reconstructed energy spectra for a $27 \mathrm{M}_{\odot}$ core-collapse $\mathrm{SN}$. The result is gained after one unfolding iteration $(k=1)$.

\section{References}

[1] H.-T. Janka, Neutrino Emission from Supernovae, in Handbook of Supernovae, edited by A. Alsabti and P. Murdin, p. 1575, Springer, 2017 [astro-ph/1702 .08713].

[2] K. Scholberg, Supernova Neutrino Detection, Annu. Rev. Nucl. Part. Sci. 62 (2012), 81-103 [astro-ph/1205.6003].

[3] JUNO Collaboration, F. An et al., Neutrino Physics with JUNO, JPG 43 (2016), 030401 [physics/1507.05613].

[4] C. Martellini et al., Towards a reconstruction of Supernova Neutrino Spectra in JUNO, EPJ Web of Conferences 209 (2019), 01012.

[5] JUNO Collaboration, T. Lin et al., Parallelized JUNO simulation software based on SNiPER, JPCS 1085 (2018), 032048 [physics/1710.07150].

[6] H.-T. Janka et al., https://wwwmpa.mpa-garching.mpg.de/ccsnarchive/archive.html, (2020).

[7] A. Oralbaev et al., The inverse beta decay: a study of cross section, JPCS, 675 (2016), 012003.

[8] M. Yang et al., Measurement of proton quenching in a LAB based liquid scintillator, Radiat. Detect. Technol. Methods. 3 (2019), 3 [physics/1809.03813].

[9] D’Agostini, Giulio, A multidimensional unfolding method based on Bayes' Theorem, NIM A 362 (1995), 487-498. 